\title{
Resistencia de Amaranthus quitensis a imazetapir y clorimurón-etil(1)
}

\author{
Daniel Tuesca(2) y Luisa Nisensohn ${ }^{(2)}$
}

\begin{abstract}
Resumen - El objetivo de este trabajo fue establecer la existencia de biotipos de A. quitensis H.B.K. resistentes a imazetapir y clorimurón-etil. Se utilizaron semillas recolectadas en las localidades de Zavalla, General Baldissera, Marcos Juárez y Las Rosas. Las dosis de herbicidas utilizadas fueron $1 / 8 \mathrm{x}, 1 / 4 \mathrm{x}, 1 / 2 \mathrm{x}, 1 \mathrm{x}, 5 \mathrm{x}, 10 \mathrm{x}$ y $20 \mathrm{x}$ de la dosis de uso recomendada. Se determinó la mortalidad y biomasa de las plantas tratadas. Se calcularon las dosis de herbicidas requeridas para reducir en un $50 \%$ la biomasa de las plantas de la maleza $\left(\mathrm{GR}_{50}\right)$ y se estimó la relación entre $\mathrm{GR}_{50}$ del biotipo resistente y $\mathrm{GR}_{50}$ del susceptible (factor de resistencia). El biotipo Zavalla resultó muy susceptible; con la mitad de la dosis de uso de ambos herbicidas la mortalidad fue $95 \%$. El biotipo General Baldissera presentó resistencia cruzada; con dosis 20 veces superiores a las recomendadas, la mortalidad fue $57,5 \%$ y $20 \%$ para imazetapir y clorimurón-etil, respectivamente. El factor de resistencia de este biotipo respecto a Zavalla fue 165 y 246 para imazetapir y clorimurón-etil, respectivamente. Los biotipos Las Rosas y Marcos Juárez fueron resistentes a imazetapir y sin embargo resultaron muy susceptibles a clorimurón-etil. Las diferencias en los patrones de resistencia estarían asociadas con distintos niveles de presión de selección en las poblaciones analizadas.
\end{abstract}

Términos para índice: biotipos, malezas, resistencia a los herbicidas.

Resistance of Amaranthus quitensis to imazethapyr and clhorimuron-ethyl

\begin{abstract}
The objective was to determine the magnitude of resistance of A. quitensis H.B.K. biotypes to imazethapyr and chlorimuron-ethyl. Weed biotypes were collected at Zavalla, General Baldissera, Marcos Juárez y Las Rosas. The herbicides were applied at 1/8x, 1/4x, 1/2x, 1x, 5x, 10x and 20x the suggested rate. Weed mortality and reduction of biomass related with untreated plants were evaluated. Herbicides concentrations required to reduce growth by $50 \%\left(\mathrm{GR}_{50}\right)$ were determined. Resistance ratio $\left(\mathrm{GR}_{50 \text { resistant }} / \mathrm{GR}_{50}\right.$ susceptible $)$ was calculated to indicate the degree of resistance. Zavalla showed high susceptibility, with $1 / 2$ of the suggested rate of both herbicides mortality reached $95 \%$. General Baldissera was cross-resistant; with rates 20 times superior to the recommended field use rate mortality was $57.5 \%$ and $20 \%$ for imazethapyr and clhorimuron-ethyl, respectively. Resistance ratios of this biotype compared with Zavalla were 165 and 246 for imazethapyr and chlorimuronethyl, respectively. Las Rosas y Marcos Juárez were resistant to imazethapyr but showed high susceptibility to chlorimuron-ethyl. The resistance patterns showed by the biotypes could be associated with differences in the selection pressure at each location.
\end{abstract}

Index terms: biotypes, weeds, herbicide resistance

\section{Introducción}

El incremento en la producción de cultivos ocurrido en la última década estuvo asociado estrechamente con el uso de herbicidas como método prin-

\footnotetext{
(1) Aceptado para publicación en 28 de junio de 2000

(2) Universidad Nacional de Rosario, Fac. Ciencias Agrarias, CC 14 (2123) Zavalla, Santa Fe, Argentina

E-mail: dtuesca@agatha.unr.edu.ar, lnisen@arnet.com.ar
}

cipal para el control de malezas (Schmidt \& Pannell, 1996).

Sin embargo, la presión de selección generada por el uso repetido de herbicidas produjo una rápida evolución hacia poblaciones de malezas resistentes (Maxwell \& Mortimer, 1994). La resistencia se define como la capacidad heredada que posee una especie para sobrevivir a la dosis de un herbicida a la cual la población original era susceptible. Este fenómeno ha ocasionado pérdidas sustanciales en 
los rendimientos de cultivos, haciendo necesario el desarrollo de nuevas estrategias de manejo de malezas (Schmidt \& Pannell, 1996).

El número de especies de malezas resistentes a herbicidas se ha incrementado en forma significativa en los últimos años (Warwick, 1991; Shaner, 1995).

Las sulfonilureas y las imidazolinonas se encuentran entre los grupos de herbicidas que actúan bloqueando la síntesis de aminoácidos esenciales por inhibición de la enzima acetolactato sintetasa (ALS). Los herbicidas que pertenecen a estos grupos poseen alta eficacia, baja toxicidad en mamíferos, amplio espectro de selectividad y sus dosis de uso son relativamente bajas. Debido a estas características su empleo está muy extendido y, como consecuencia de ello, la resistencia a herbicidas pertenecientes a estos grupos se ha constituído en los últimos años en un serio problema. La selección de biotipos resistentes a estos herbicidas puede ocurrir en períodos relativamente cortos, de tres a siete años (Saari et al., 1994; Lovell et al., 1996).

La aplicación repetida de estos herbicidas ha favorecido la selección de biotipos resistentes en diferentes especies de malezas del género Amaranthus entre las que se pueden citar A. blitoides, A. retroflexus (Saari et al., 1994), A. palmeri, A. rudis (Horak \& Peterson, 1995), A. hybridus y A. lividus (Manley et al., 1996). La selección de biotipos resistentes en este género podría ocurrir con bastante facilidad debido a su gran susceptibilidad a estos herbicidas y a su elevada fecundidad (Faccini \& Nisensohn, 1994; Manley et al., 1996).

En Argentina, A. quitensis H.B.K. es una de las malezas anuales más importantes en los cultivos de verano y causa pérdidas considerables de rendimiento por competencia (Leguizamón et al., 1994).

En los cultivos de soja del área pampeana argentina es habitual el empleo sistemático de herbicidas del grupo de las imidazolinonas y sulfonilureas. En esta zona se han detectado numerosos casos de baja susceptibilidad a estos herbicidas en poblaciones de A. quitensis. Se ha sugerido que estas fallas en el control podrían estar asociadas con la selección de biotipos resistentes a estos herbicidas.

El objetivo de este trabajo fue determinar la existencia de biotipos de $A$. quitensis resistentes a her- bicidas del grupo de las imidazolinonas y sulfonilureas.

\section{Material y Métodos}

Se obtuvieron semillas de $A$. quitensis en campos en los que se habían detectado fallas en el control de esta maleza al emplearse herbicidas de los grupos imidazolinonas y sulfonilureas. Asimismo, se recolectaron semillas de otra población (Zavalla) en la cual no se observaron deficiencias en el control en los últimos años. Los biotipos sospechosos de presentar resistencia fueron Marcos Juárez, General Baldissera y Las Rosas.

Los cultivos estivales y los herbicidas utilizados durante los últimos cinco años en las distintas localidades se observan en el Cuadro 1

El ensayo se llevó a cabo en el Campo Experimental J. F. Villarino de la Facultad de Ciencias Agrarias de la Universidad Nacional de Rosario ubicado en la localidad de Zavalla, Santa Fe, Argentina a $33^{\circ} 0^{\prime}$ S y $60^{\circ} 53^{\prime} \mathrm{W}$.

Se sembraron 60 semillas de cada biotipo en recipientes plásticos de $250 \mathrm{~mm}$ de diámetro que contenían una mezcla de tierra, arena y perlita en una relación volumétrica de $70 \%: 20 \%: 10 \%$, previamente esterilizada con bromuro de metilo. Estos recipientes permanecieron en invernáculo durante los primeros siete días y posteriormente se ubicaron en el exterior. Luego de la emergencia se estableció mediante raleo una densidad de diez plantas de la maleza en cada recipiente.

En el momento en que las plantas tenían cuatro a seis hojas (50-60 mm de altura) se aplicaron los distintos tratamientos.

Los herbicidas y las dosis empleadas fueron: imazetapir a $1,25,2,5,5,0,10,0,50,0,100,0$ y $200,0 \mathrm{mg} / \mathrm{m}^{2}$ de i.a. y clorimurón-etil a $0,125,0,250,0,5,1,0,5,0,10,0 \mathrm{y}$ $20,0 \mathrm{mg} / \mathrm{m}^{2}$ de i.a. Estas dosis representan $1 / 8 \mathrm{x}, 1 / 4 \mathrm{x}$, $1 / 2 \mathrm{x}, 1 \mathrm{x}, 5 \mathrm{x}, 10 \mathrm{x}$ y $20 \mathrm{x}$ de la dosis de uso recomendada, respectivamente

Los herbicidas fueron aplicados utilizando una mochila de presión constante con fuente de $\mathrm{CO}_{2}$ equipada con pastillas XR11001. La presión fue de $241 \mathrm{kPa}$, y el caudal, de $130 \mathrm{~L} / \mathrm{ha}$

El diseño del ensayo fue completamente aleatorizado con cuatro repeticiones. Dentro de cada biotipo el análisis se realizó comparando el efecto de distintas dosis de un mismo herbicida

A los 30 días de la aplicación se registró el número de plantas sobrevivientes de cada biotipo de la maleza en los distintos tratamientos y se calculó el porcentaje de mortalidad. Estas plantas fueron cosechadas y colocadas en estufa a $80^{\circ} \mathrm{C}$ durante 48 horas. Se determinó su mate- 
Cuadro 1. Cultivos y herbicidas empleados en los sitios de recolección de los distintos biotipos en los diferentes años.

\begin{tabular}{|c|c|c|c|c|c|c|c|c|c|c|}
\hline \multirow[t]{2}{*}{ Biotipos } & \multicolumn{2}{|r|}{1993} & \multicolumn{2}{|c|}{1994} & \multicolumn{2}{|r|}{1995} & \multicolumn{2}{|c|}{1996} & \multicolumn{2}{|r|}{1997} \\
\hline & Cultivo & Herbicida & $\overline{\text { Cultivo }}$ & Herbicida & Cultivo & Herbicida & Cultivo & Herbicida & Cultivo & Herbicida \\
\hline Zavalla & Soja & Imazetapir & Maíz & Atrazina & Soja & Bentazón + 2,4-DB & Maíz & Atrazina & Soja & Bentazón + 2,4-DB \\
\hline G. Baldissere & Maíz & Nicosulfurón & Soja & Imazetapir & Soja & Clorimurón-etil & Soja & Imazetapir & Soja & Imazetapir \\
\hline Las Rosas & Soja & Imazetapir & Soja & Imazetapir & Soja & Imazetapir & Maíz & Atrazina & Soja & Imazetapir \\
\hline Marcos Juarez & Soja & Imazetapir & Soja & Imazetapir & Soja & Imazetapir & Soja & Imazetapir & Soja & Imazetapir \\
\hline
\end{tabular}

ria seca y con estos valores se calculó el porcentaje de biomasa respecto al testigo no tratado.

Tanto los datos de mortalidad como de materia seca se transformaron mediante arc sen $(x+1)^{1 / 2}$ y se analizaron utilizando ANOVA. Las medias se compararon empleando el test de Mínima Diferencia Significativa con una probabilidad $(\mathrm{p} \leq 0,05)$.

Mediante análisis de regresión se determinaron las funciones que relacionaban las dosis de herbicida con la materia seca de las plantas tratadas. A partir de las ecuaciones de regresión se calcularon las dosis de herbicidas requeridas para reducir en un $50 \%$ la biomasa de las plantas de la maleza $\left(\mathrm{GR}_{50}\right)$. El grado de resistencia de las poblaciones analizadas se calculó utilizando el factor de resistencia que es el cociente entre el $\mathrm{GR}_{50}$ del biotipo resistente y el $\mathrm{GR}_{50}$ del biotipo susceptible.

\section{Resultados y Discusión}

Al analizar la mortalidad de los diferentes biotipos de $A$. quitensis se observó que en el tratamiento donde se aplicaron $5 \mathrm{mg} / \mathrm{m}^{2}$ de i.a. de imazetapir, equivalente a la mitad de la dosis normal de uso, el biotipo Zavalla resultó muy afectado y la mortalidad fue del $95 \%$ mientras que en el resto de los biotipos considerados la mortalidad fue nula (Cuadro 2). Un comportamiento similar se observó tanto al emplearse $10 \mathrm{mg} / \mathrm{m}^{2}$ de i.a. (dosis de marbete) como $50 \mathrm{mg} / \mathrm{m}^{2}$ de i.a. que representa cinco veces la dosis recomendada. En el biotipo General Baldissera sólo se alcanzó el 57,5\% de mortalidad cuando se empleó una dosis 20 veces superior a la dosis normal de uso.

En el tratamiento con clorimurón-etil a la dosis de $1 \mathrm{mg} / \mathrm{m}^{2}$ de i.a. que corresponde a la dosis recomendada, los biotipos Zavalla, Las Rosas y Marcos Juárez resultaron muy susceptibles y presentaron una mortalidad cercana al $100 \%$. El biotipo General Baldissera presentó un comportamiento similar al observado para imazetapir y la mortalidad sólo fue del $20 \%$ con una dosis 20 veces superior a la dosis de uso.

$\mathrm{Al}$ analizar la materia seca de las plantas sobrevivientes en los tratamientos con imazetapir (Cuadro 3) el biotipo Zavalla mostró una marcada reducción en su biomasa de aproximadamente $80 \%$ al emplearse $1,25 \mathrm{mg} / \mathrm{m}^{2}$ de i.a., que corresponde a $1 / 8$ de la dosis de uso. El biotipo menos susceptible fue General Baldissera, ya que con una dosis 20 veces superior a la dosis de marbete su biomasa se redujo sólo $54 \%$.

Al utilizar clorimurón-etil a la dosis recomendada $\left(1 \mathrm{mg} / \mathrm{m}^{2}\right.$ de i.a.) los biotipos Zavalla, Las Rosas y Marcos Juárez resultaron susceptibles y la biomasa de las plantas sobrevivientes se redujo en más del $95 \%$. Por el contrario, el biotipo General Baldissera mostró muy baja susceptibilidad ya que su biomasa se redujo $58 \%$ con dosis de $20 \mathrm{mg} / \mathrm{m}^{2}$ de i.a.

Las ecuaciones que describen la relación entre la biomasa de las plantas tratadas y las dosis del herbicida generaron funciones con coeficientes de regresión significativos (Cuadro 4).

En el biotipo Zavalla el GR $_{50}$ se alcanzó con valores muy inferiores a la dosis normal de uso de $10 \mathrm{mg} / \mathrm{m}^{2}$ de i.a. (Cuadro 5). Los biotipos General Baldissera, Las Rosas y Marcos Juárez presentaron resistencia al herbicida imazetapir, hecho que se observa en los elevados valores de $\mathrm{GR}_{50}$. Estos biotipos mostraron diferentes grados de resistencia, siendo General Baldissera el biotipo más resistente, ya que fue necesario aumentar 19 veces la dosis normal de uso para reducir su biomasa a la mitad.

Este patrón de comportamiento difirió al emplearse clorimurón-etil. Además de Zavalla, los biotipos Las Rosas y Marcos Juárez resultaron muy susceptibles y la dosis necesaria para reducir el $50 \%$ de la biomasa fue inferior al $10 \%$ de la dosis normal de uso $\left(1 \mathrm{mg} / \mathrm{m}^{2}\right.$ de i.a.). Por el contrario, en el caso 
Cuadro 2. Porcentaje de mortalidad de los distintos biotipos de Amaranthus quitensis a los 30 días de la aplicación de los tratamientos postemergentes ${ }^{(1)}$.

\begin{tabular}{|c|c|c|c|c|c|}
\hline Herbicida & Dosis ( $\mathrm{mg} / \mathrm{m}^{2}$ de i.a.) & Zavalla & G. Baldissera & L. Rosas & M. Juárez \\
\hline \multirow[t]{8}{*}{ Imazetapir } & 0,00 & $0 \mathrm{c}$ & $0,0 \mathrm{~b}$ & $0,0 \mathrm{~d}$ & $0 \mathrm{c}$ \\
\hline & 1,25 & $10 \mathrm{~b}$ & $0,0 \mathrm{~b}$ & $0,0 \mathrm{~d}$ & $0 \mathrm{c}$ \\
\hline & 2,50 & $10 \mathrm{~b}$ & $0,0 \mathrm{~b}$ & $0,0 \mathrm{~d}$ & $0 \mathrm{c}$ \\
\hline & 5,00 & $95 \mathrm{a}$ & $0,0 \mathrm{~b}$ & $0,0 \mathrm{~d}$ & $0 \mathrm{c}$ \\
\hline & 10,00 & $100 \mathrm{a}$ & $0,0 \mathrm{~b}$ & $10,0 \mathrm{~cd}$ & $0 \mathrm{c}$ \\
\hline & 50,00 & $100 \mathrm{a}$ & $5,0 \mathrm{~b}$ & $15,0 \mathrm{c}$ & $10 \mathrm{c}$ \\
\hline & 100,00 & $100 \mathrm{a}$ & $5,0 \mathrm{~b}$ & $60,0 \mathrm{~b}$ & $25 b$ \\
\hline & 200,00 & $100 \mathrm{a}$ & $57,5 \mathrm{a}$ & $92,5 \mathrm{a}$ & $77 \mathrm{a}$ \\
\hline \multirow[t]{8}{*}{ Clorimurón-etil } & 0,000 & $0,0 \mathrm{c}$ & $0,0 \mathrm{c}$ & $0,0 \mathrm{~d}$ & $0,0 \mathrm{c}$ \\
\hline & 0,125 & $15,0 \mathrm{~b}$ & $0,0 \mathrm{c}$ & $20,0 \mathrm{c}$ & $17,5 b$ \\
\hline & 0,250 & $17,5 b$ & $0,0 \mathrm{c}$ & $22,5 \mathrm{c}$ & $20,0 b$ \\
\hline & 0,500 & $95,0 \mathrm{a}$ & $0,0 \mathrm{c}$ & $72,5 b$ & $95,0 \mathrm{a}$ \\
\hline & 1,000 & $98,0 \mathrm{a}$ & $5,0 \mathrm{bc}$ & $95,0 \mathrm{a}$ & $98,0 \mathrm{a}$ \\
\hline & 5,000 & $100,0 \mathrm{a}$ & $12,5 \mathrm{ab}$ & $100,0 \mathrm{a}$ & $100,0 \mathrm{a}$ \\
\hline & 10,000 & $100,0 \mathrm{a}$ & $15,0 \mathrm{a}$ & $100,0 \mathrm{a}$ & $100,0 \mathrm{a}$ \\
\hline & 20,000 & $100,0 \mathrm{a}$ & $20,0 \mathrm{a}$ & $100,0 \mathrm{a}$ & $100,0 \mathrm{a}$ \\
\hline
\end{tabular}

${ }^{(1)}$ En cada biotipo, letras distintas, indican diferencias significativas $(p \leq 0,05)$ entre las dosis de un mismo herbicida según el test de Mínima Diferencia Significativa.

Cuadro 3. Materia seca de las plantas sobrevivientes de Amaranthus quitensis expresado como porcentaje del testigo de cada biotipo a los 30 días del tratamiento(1).

\begin{tabular}{lccccc}
\hline Herbicida & Dosis $\left(\mathrm{mg} / \mathrm{m}^{2}\right.$ de i.a. $)$ & Zavalla & G. Baldissera & L. Rosas & M. Juárez \\
\hline Imazetapir & 1,25 & $22,8 \mathrm{a}$ & $100,0 \mathrm{a}$ & $100,0 \mathrm{a}$ & $100,0 \mathrm{a}$ \\
& 2,50 & $9,6 \mathrm{~b}$ & $100,0 \mathrm{a}$ & $100,0 \mathrm{a}$ & $100,0 \mathrm{a}$ \\
& 5,00 & $4,6 \mathrm{c}$ & $88,7 \mathrm{~b}$ & $98,2 \mathrm{a}$ & $80,6 \mathrm{~b}$ \\
& 10,00 & $0,0 \mathrm{~d}$ & $86,1 \mathrm{bc}$ & $82,5 \mathrm{~b}$ & $77,8 \mathrm{~b}$ \\
& 50,00 & $0,0 \mathrm{~d}$ & $80,2 \mathrm{bc}$ & $66,6 \mathrm{c}$ & $70,8 \mathrm{~b}$ \\
& 100,00 & $0,0 \mathrm{~d}$ & $78,2 \mathrm{c}$ & $16,4 \mathrm{~d}$ & $67,0 \mathrm{~b}$ \\
\hline Clorimurón-etil & 200,00 & $0,0 \mathrm{~d}$ & $45,9 \mathrm{~d}$ & $0,5 \mathrm{e}$ & $25,2 \mathrm{c}$ \\
& 0,125 & $35,0 \mathrm{a}$ & $100,0 \mathrm{a}$ & $29,2 \mathrm{a}$ & $28,3 \mathrm{a}$ \\
& 0,25 & $28,0 \mathrm{a}$ & $100,0 \mathrm{a}$ & $23,2 \mathrm{a}$ & $22,3 \mathrm{~b}$ \\
& 0,5 & $12,6 \mathrm{~b}$ & $83,9 \mathrm{~b}$ & $9,6 \mathrm{~b}$ & $8,8 \mathrm{c}$ \\
& 1,000 & $3,7 \mathrm{bc}$ & $68,9 \mathrm{c}$ & $3,7 \mathrm{~b}$ & $2,2 \mathrm{~d}$ \\
& 5,000 & $0,0 \mathrm{c}$ & $62,3 \mathrm{c}$ & $0,0 \mathrm{~b}$ & $0,0 \mathrm{e}$ \\
& 10,000 & $0,0 \mathrm{c}$ & $52,6 \mathrm{~d}$ & $0,0 \mathrm{~b}$ & $0,0 \mathrm{e}$ \\
\hline
\end{tabular}

${ }^{(1)}$ En cada biotipo, letras distintas, indican diferencias significativas $(\mathrm{p} \leq 0,05)$ entre las dosis de un mismo herbicida según el test de Mínima Diferencia Significativa.

del biotipo General Baldissera se observó resistencia y fue necesario aumentar diez veces la dosis normal de uso para reducir en un 50\% la biomasa de las plantas tratadas.

$\mathrm{Al}$ analizar el factor de resistencia se observa que en el biotipo General Baldissera la relación entre la dosis requerida para disminuir el $50 \%$ de su biomasa respecto a la del biotipo susceptible (Zavalla) fue de 165 y de 246 para imazetapir y clorimurón-etil, respectivamente (Cuadro 5).

De estos resultados se deduce que los patrones de resistencia difieren entre los distintos biotipos. Mientras las poblaciones de Marcos Juárez y Las Rosas solamente resultaron resistentes a imazetapir, el biotipo General Baldissera mostró resistencia cruzada tanto a imazetapir como a clorimurón-etil. Este 
Cuadro 4. Ecuaciones de regresión utilizadas para el cálculo de los valores de $\mathrm{GR}_{50}$ de los distintos biotipos. $\mathrm{y}=$ biomasa expresada como porcentaje del testigo no tratado; $\mathrm{x}=$ dosis del herbicida en $\mathrm{mg} / \mathrm{m}^{2}$ de i.a.

\begin{tabular}{llc}
\hline Biotipo & Ecuación & $\mathrm{R}^{2}$ \\
\hline \multirow{2}{*}{ Zavalla } & \multicolumn{1}{c}{ Imazetapir } \\
General Baldissera & $\mathrm{y}=\mathrm{e}^{(5,45-1,34 \mathrm{x})}$ & 0,91 \\
Las Rosas & $\mathrm{y}=96,01-0,24 \mathrm{x}$ & 0,83 \\
Marcos Juárez & $\mathrm{y}=92,97-0,54 \mathrm{x}$ & 0,88 \\
\hline \multirow{2}{c}{ Clorimurón-etil } \\
Zavalla & $\mathrm{y}=\mathrm{e}^{(4,06-2,66 \mathrm{x})}$ & 0,84 \\
General Baldissera & $\mathrm{y}=88,94-2,75 \mathrm{x}$ & 0,99 \\
Las Rosas & $\mathrm{y}=\mathrm{e}^{(4,07-3,11 \mathrm{x})}$ & 0,71 \\
Marcos Juárez & $\mathrm{y}=\mathrm{e}^{(3,97-3,10 \mathrm{x})}$ & 0,99 \\
& & 0,99 \\
\hline
\end{tabular}

fenómeno de resistencia a herbicidas de grupos distintos que comparten el mismo modo de acción ha sido encontrado por otros autores en especies de este género (Lovell et al., 1996; Gaeddert et al., 1997; Hinz \& Owen, 1997; Sprague et al., 1997).

Una de las características determinantes en la evolución de biotipos resistentes es la presión de selección ejercida por los herbicidas. Del análisis de los resultados surge que las dosis comerciales de los herbicidas analizados en este trabajo son excesivamente altas para controlar los biotipos susceptibles. Esto ocurre debido a que normalmente las dosis comerciales se establecen teniendo en cuenta la cantidad necesaria de producto para el control de las especies de malezas menos sensibles. Así, la presión de selección impuesta sobre $A$. quitensis ha sido muy alta, lo que probablemente influyó en la aparición de biotipos resistentes.

Adicionalmente, estos grupos de herbicidas presentan residualidad, lo que resulta en un aumento de la presión de selección hacia biotipos resistentes. Esto es particularmente importante en el caso de A. quitensis, debido a que la emergencia de plántulas ocurre en cohortes (Faccini \& Nisensohn, 1994).

La presión de selección de biotipos resistentes se incrementa con el empleo de dosis elevadas de herbicidas. En este sentido, es de esperar que en los sistemas de siembra directa, en los cuales no sólo es alta la frecuencia de aplicación de herbicidas sino también sus dosis (Mitidieri, 1989), la probabilidad de generar biotipos resistentes sea mayor en compa-
Cuadro 5. Dosis de herbicidas ( $\mathrm{mg} / \mathrm{m}^{2}$ de i.a.) requeridas para reducir $50 \%$ la biomasa de los biotipos analizados $\left(\mathrm{GR}_{50}\right)$ de Amaranthus quitensis.

\begin{tabular}{|c|c|c|c|}
\hline Biotipo & $\mathrm{GR}_{50 \text { susc }}$ & $\mathrm{GR}_{50 \text { res }}$ & $\mathrm{R} / \mathrm{S}^{(1)}$ \\
\hline & \multicolumn{3}{|c|}{ Imazetapir } \\
\hline Zavalla & 1,152 & & \\
\hline Baldissera & & 190,59 & 165,44 \\
\hline Las Rosas & & 84,2 & 73,09 \\
\hline \multirow[t]{2}{*}{ Marcos Juárez } & & 129,8 & 112,67 \\
\hline & \multicolumn{3}{|c|}{ Clorimurón-etil } \\
\hline Zavalla & 0,057 & & \\
\hline Baldissera & & 14,12 & 246,49 \\
\hline Las Rosas & 0,052 & & \\
\hline Marcos Juarez & 0,022 & & \\
\hline
\end{tabular}

ración a sistemas de labranza convencional. Por otra parte, en los sistemas no laboreados se espera que la difusión de la resistencia sea más rápida, ya que al no existir remoción del suelo se reduciría la probabilidad de que las poblaciones de semillas susceptibles amortigüen la presión de selección impuesta.

En los biotipos analizados, las diferencias observadas en los patrones de resistencia estarían asociadas a distintas presiones de selección. Los biotipos General Baldissera, Las Rosas y Marcos Juárez corresponden a sistemas donde la siembra directa de cultivos de verano, y las aplicaciones sistemáticas de herbicidas en altas dosis son una práctica habitual desde hace casi diez años. Por el contrario, el biotipo Zavalla proviene de un lote donde predomina el sistema de laboreo convencional y el control químico se realiza con dosis reducidas de herbicidas. Asimismo en este sitio la frecuencia de uso de herbicidas inhibidores de la ALS fue significativamente menor que en los otros. Estos resultados confirman la importancia de utilizar en forma alternada herbicidas con diferentes modos de acción para reducir el riesgo de aparición de resistencia.

\section{Conclusiones}

1. Los biotipos analizados muestran distintos patrones de resistencia frente a los herbicidas imazetapir y clorimurón-etil.

2. Zavalla es muy susceptible a ambos herbicidas aún a dosis significativamente menores que las dosis recomendadas en el marbete. 
3. Las Rosas y Marcos Juárez son resistentes a imazetapir y sin embargo resultan muy susceptibles a la aplicación de clorimurón-etil.

4. General Baldissera presenta resistencia cruzada a ambos herbicidas.

\section{Referencias}

FACCINI, D. E.; NISENSOHN, L. A. Dinámica de la población de yuyo colorado (Amaranthus quitensis H.B.K.): influencia de los tratamientos químicos y mecánicos en un cultivo de soja. Pesquisa Agropecuária Brasileira, Brasília, v. 29, n. 7, p. 1041-1050, jul. 1994.

GAEDDERT, J. W.; PETERSON, D. E.; HORAK, M. J. Control and cross-resistance of an acetolactate synthase inhibitor-resistant palmer amaranth (Amaranthus palmeri) biotype. Weed Technology, Champaign, v. 11, n. 1, p. 132-137, 1997

HINZ, J. R. R.; OWEN, M. D. K. Acetolactate synthase resistance in a common waterhemp (Amaranthus rudis) population. Weed Technology, Champaign, v. 11, n. 1, p. 13-18, 1997.

HORAK, M. J.; PETERSON, D. E. Biotypes of palmer amaranth (Amaranthus palmeri) and common waterhemp (Amaranthus rudis) are resistant to imazethapyr and thifensulfuron. Weed Technology, Champaign, v. 9, n. 1, p. $192-195,1995$

LEGUIZAMÓN, E.; FACCINI, D.; NISENSOHN, L.; PURICELLI, E.; MITIDIERI,A.; LOPEZ, J.; RAINERO, H.; PAPA, J.; ROSSI, R.; CEPEDA, S.; PONSA, J.; MORENO, R.; FAYA, L. Funciones de daño y cálculo de pérdidas por malezas en el cultivo de soja. Pergamino : Instituto Nacional de Tecnología Agropecuaria, 1994. p. 1-19. (INTA. Informe Técnico, 296).

LOVELL, S. T.; WAX, L. M.; HORAK, M. J.; PETERSON, D. E. Imidazolinone and sulfonylurea resistance in a biotype of common waterhemp
(Amaranthus rudis). Weed Science, Champaign, v. 44, n. 4, p. 789-794, 1996

MANLEY, B. S.; WILSON, H. P.; HINES, T. E. Smooth pigweed (Amaranthus hybridus) and livid amaranth (A. lividus) response to several imidazolinone and sulfonylurea herbicides. Weed Technology, Champaign, v. 4, n. 4 , p. $835-841,1996$.

MAXWELL, B. D.; MORTIMER, A. M. Selection for herbicide resistance. In: POWLES, S. B.; HOLTUM, J. A. M. (Ed.). Herbicide resistance in plants: biology and biochemistry. Boca Raton : Lewis, 1994. p. 1-25.

MITIDIERI,A. El control químico de las malezas en soja en Argentina. In: WORLD SOYBEAN RESEARCH CONFERENCE, 4., 1989, Buenos Aires. Proceedings.. Buenos Aires : Asociación Argentina de la Soja, 1989. p. 2117-2122.

SAARI, L. L.; COTTERMAN, J. C.; THILL, D. C Resistance to acetolactate synthase inhibiting herbicides. In: POWLES, S. B.; HOLTUM, J. A. M. (Ed.). Herbicide resistance in plants: biology and biochemistry. Boca Raton : Lewis, 1994. p. 83-139.

SCHMIDT, C. P.; PANNELL, D. J. Economic issues in management of herbicide-resistant weeds. Review of Marketing and Agricultural Economics, Melbourne, v. 64, n. 3 , p. $301-308,1996$

SHANER, D. L. Herbicide resistance: where are we? How did we get here? Where are we going? Weed Technology, Champaign, v. 9, n. 4, p. 850-856, 1995.

SPRAGUE, C. L.; STOLLER, E. W.; WAX, L. M.; HORAK, M. J. Palmer amaranth (Amaranthus palmeri) and common waterhemp (Amaranthus rudis) resistance to selected ALS-inhibiting herbicides. Weed Science, Champaign, v. 45, n. 2, p. 192-197, 1997.

WARWICK, S. I. Herbicide resistance in weedy plants: physiology and population biology. Annual Review of Ecology and Systematics, Palo Alto, v. 22, n. 1, p. 95114, 1991 